\title{
le rôle du géotechnicien en expertise judiciaire
}

\author{
G. SANGLERAT \\ Professeur de mécanique des sols et fondations à l'école centrale de Lyon \\ Expert agréé par la cour de cassation
}

T.R. SANGLERAT

Ingénieur I.N.S.A., Master of Science (Norwestern University)

\section{INTRODUCTION}

Dans la pratique, les intervenants dans l'acte de construire ou dans le cours d'une expertise s'interrogent souvent pour savoir s'il est nécessaire de recourir ou non à un spécialiste géotechnicien ou géologue pour traiter des problèmes de fondations ou de stabilité de terrain.

Dans le cas de la conception de fondations, la réponse est très simple, l'intervention du géotechnicien est obligatoire du fait de la nouvelle loi Spinetta. Tous les participants à l'acte de construire doivent être assurés; or l'ingénieur de structures qui, très souvent, dans le passé, définissait, seul ou avec le bureau de contrôle, les fondations (types, niveaux, géométrie, contraintes et tassements admissibles), n'est plus assuré pour ce genre de prestations.

Il est donc indispensable que le maître d'ouvrage, le maître d'œuvre, le bureau d'études ou l'entreprise sollicitent au moment de la conception des fondations, l'intervention d'un géotechnicien qualifié et de surcroît correctement assuré pour ses responsabilités décennale et civile. Le bureau de contrôle doit vérifier par ailleurs que l'étude géotechnique a été correctement conduite à partir de reconnaissances suffisantes pour être représentatives du site étudié.
Par contre, lorsqu'il s'agit d'une expertise concernant des désordres nouveaux survenus dans un ouvrage existant, récent ou ancien, il arrive souvent que des experts généralistes aient à prendre des décisions liées à des problèmes de mécanique des sols et fondations et là, l'intervention d'un spécialiste n'est pas, à ce jour, légalement obligatoire.

Ces généralistes peuvent-ils alors prendre seuls leurs décisions, ou au contraire doivent-ils faire appel à un spécialiste (géotechnicien ou géologue)?

Examinons pourquoi ils doivent se poser cette question et, dans quels cas ils doivent y répondre par l'affirmative et quels sont les critères qui devraient guider leur choix.

\section{UTILITÉ DE L'INTERVENTION D'UN GÉOTECHNICIEN}

Nous distinguerons deux cas: soit l'expertise amiable diligentée le plus souvent par des experts de compagnie d'assurances, soit l'expertise judiciaire où interviennent, outre l'expert désigné par le tribunal, les experts des parties, ou de leurs compagnies d'assu- 
rances. Chacun de ces experts doit se demander s'il faut faire appel à un «sapiteur» géotechnicien ou géologue.

\subsection{Cas de l'expertise amiable}

L'exemple le plus courant est celui de l'expert de compagnie d'assurances qui est, le plus souvent, un généraliste traitant des sinistres d'origines extrêmement diverses. Néanmoins, il doit définir aussi bien dans l'intérêt de l'assuré que de la compagnie qu'il réprésente, s'il doit prendre en charge ou non les conséquences du sinistre examiné.

Souvent une décision très rapide doit être prise, d'abord pour limiter l'accroissement des désordres observés en stoppant les causes de ceux-ci, ensuite, en raison de l'érosion monétaire; une estimation du préjudice rapide pourra être plus intéressante sur le plan économique pour la compagnie d'assurances, tout en donnant pleinement satisfaction à l'assuré ou aux tiers ayant subi les préjudices.

Mais pour prendre une décision pertinente, qui ne risque pas de se voir ultérieurement contredite au cours d'une expertise judiciaire dans le cas où l'affaire serait portée devant la juridiction compétente, il importe de connaître l'origine et la cause exacte des désordres pour savoir si le sinistre relève des garanties accordées par la police d'assurance ou non.

Une décision hative à ce sujet, qui se révélerait erronée par la suite, pourrait avoir des conséquences considérables. En outre, si un accident corporel se produisait parce que des travaux conservatoires d'urgence n'ont pas été prescrits et exécutés en temps utile, la position de l'expert pourrait être très inconfortable.

\subsection{Cas de l'expertise judiciaire}

On se retrouve généralement devant le tribunal lorsque précisément l'expertise amiable mentionnée ci-dessus n'a pu aboutir pour différentes raisons. L'expérience montre qu'assez souvent cet échec de la tentative amiable résulte du fait que le problème technique a été mal compris, ou mal apprécié, par les experts des compagnies d'assurances des parties en cause.

Mettons-nous alors à la place de l'expert judiciaire à qui l'on soumet un certain nombre d'opinions contradictoires, justifiées par des avis techniques plus ou moins étayés ou convaincants, parmi lesquelles il doit déterminer celles correspondant à la réalité. Il peut d'ailleurs arriver que tous les avis formulés soient ou erronés ou exprimés d'une manière très partiale, voire même tendancieuse. Le problème technique doit alors être repris dans son ensemble.

Souvent, l'expert judiciaire est lui aussi un généraliste. Il a cependant pour mission de trouver les causes réelles des désordres et ce, pour différentes raisons:

- Pour déterminer s'il y a urgence ou non;

- Pour décider quelles sont les mesures à prescrire: simples réfections ou travaux confortatifs pour éviter l'accroissement des désordres, voire même l'effondrement de louvrage;

- Pour définir toutes les causes des désordres et préjudices avec précision afin de permettre aux magistrats de déterminer ultérieurement les responsabilités encourues.

En effet, si l'expert judiciaire n'a jamais à fixer les responsabilités, rôle qui incombe aux magistrats, la tâche de ces derniers sera facilitée si les causes sont bien décrites et correctement analysées. D'ailleurs, le plus souvent, sauf motif juridique particulier, les responsabilités découleront pratiquement des causes des désordres. D'où l'importance pour l'expert judiciaire de ne pas se tromper, car il pourrait alors conduire les magistrats à condamner des parties, quelquefois lourdement, pour des fautes qu'elles n'auraient pas commises en réalité.

L'expert judiciaire se trouvera quelquefois devant des cas difficiles où il devra prendre une décision rapide pour présenter un ouvrage et s'il se trompe, il pourra, soit prescrire des travaux confortatifs inutiles, qu'on lui reprochera par la suite, soit, au contraire laisser accroître les préjudices d'une manière considérable, ce qui est également critiquable.

En effet, il est indispensable, par exemple, en cas d'apparition de fissures de déterminer si celle-ci sont imputables à des malfaçons dans les structures (mauvaise exécution du feraillage ou du bétonnage, absence de joints de dilatation) ou, au contraire, à une mauvaise conception des fondations ou à des erreurs dans leur exécution résultant de l'ignorance des règles de l'art en mécanique des sols.

Ensuite, il importe de prévoir l'évolution dans le temps de ces désordres et tant que l'on n'aura pas déterminé exactement leurs causes réelles, il sera impossible, naturellement, de se prononcer sur le comportement de l'ouurage à court, moyen ou long terme.

Il existe également un autre cas où l'expert judiciaire, comme l'expert de compagnie d'assurances, peut se trouver devant une décision délicate à prendre. Faut-il prescrire des investigations coûteuses pour mieux cerner un problème et analyser plus correctement les causes éventuelles des désordres observés?

En effet, avant de faire exécuter des reconnaissances de sol in situ ou des essais de laboratoire sur échantillons intacts toujours coûteux et longs à obtenir, il faut être sûr que les désordres peuvent être imputables au sol et que les investigations préconisées permettront de clarifier la situation. Sinon, on risquerait d'engager des dépenses importantes pour un résultat insignifiant, que les parties ne manqueraient pas, ultérieurement, de reprocher, à juste titre, à l'expert judiciaire. Il est donc indispensable de faire appel à un géotechnicien qualifié avant de prendre de telles décisions. La plupart du temps, ce spécialiste pourra, après examen des lieux et de documents disponibles, dire si ces recherches s'avèrent indispensables, ou si elles sont, au contraire, a priori inutiles.

Précisons que si l'expert judiciaire cherchait à s'abriter derrière une abstention prudente et que les désordres 
s'aggravent il pourrait alors lui être reproché sa carence.

Naturellement, pour éviter ce genre d'inconvénients, on pourrait se demander pourquoi les magistrats ne désignent pas toujours un spécialiste des désordres à réparer?

A notre avis, il ne faut pas jeter la pierre aux magistrats, car, très souvent, les demandeurs euxmêmes, pas plus que les défendeurs, ne connaissent l'origine des désordres. C'est pourquoi il peut arriver qu'un architecte ou un ingénieur structure soient désignés par décision judiciaire et qu'il apparaisse, à l'analyse que les problèmes sont essentiellement liés à la mécanique des sols (fondations défaillantes, inobservations des règles de l'art dans la conception ou l'exécution des terrassements ou des fondations, drainage insuffisant, etc.). Il est bien évident que lorsque le sinistre a une origine claire, par exemple glissement de terrain, mur de soutènement effondré, ou défaillance manifeste des fondations, il est souhaitable alors que les magistrats désignent directement un spécialiste de mécanique des sols, et non pas un généraliste. Si nécessaire, ce géotechnicien pourra faire appel à un géologue. On évitera ainsi d'alourdir l'expertise et de retarder son bon déroulement ou même de la voir s'acheminer vers des conclusions quelquefois contestables surtout si le généraliste croit avoir compris le phénomène et qu'il se trompe, ce qui peut arriver dans des cas difficiles, car il se trouve hors du domaine de ses compétences propres.

Pour illustrer ce qui vient d'être exposé, un certain nombre de cas réellement vécus, feront comprendre quelques-uns des problèmes qui peuvent être liés à la mécanique des sols dans la pratique quotidienne de l'expertise judiciaire ou amiable, en bâtiments ou travaux publics.

\section{EXEMPLES DE CAS RÉELS}

\subsection{Tassements différentiels dans des dallages industriels}

Nous avons eu l'occasion d'exposer dans les «Cahiers de l'Expertise Judiciaire » $\mathrm{n}^{\circ} 1$, les difficultés d'une expertise judiciaire relative à des tassements différentiels de dallages industriels dont le coût de réfection a atteint 6 millions de francs. Une particularité de cette expertise, à laquelle ont participé deux géotechniciens confirmés, était que les tassements des dallages pouvaient entraîner la rupture de la charpente métallique qui soutenait les bardages extérieurs de l'usine. Nous pouvons préciser, aujourd'hui, que les réfections préconisées ont donné toutes satisfactions. Il est certain que si aucun géotechnicien n'était intervenu dans cette expertise, les problèmes rencontrés n'auraient pu être résolus correctement.

\subsection{Fissures dans un plancher en rez-de-chaussée sur terre-plein}

Sur la Côte d'Azur, dans un bâtiment d'habitation d'excellente qualité, des fissures apparurent dans le plancher du rez-de-chaussée coulé directement sur terre-plein. L'expert judiciaire, non spécialiste en mécanique des sols, attribua, sans analyses particulières, ces fissures à des tassements du sol porteur et estima qu'il était nécessaire d'injecter le sol sous les planchers. Une entreprise spécialisée exécuta pour $300000 \mathrm{~F}$ d'injections de coulis au ciment. Et malgré cette dépense importante, quelques mois plus tard, les fissures réapparurent.

A ce moment là, un ingénieur de bureau de contrôle, enfin alerté, connaissant bien les terrains de la région pu affirmer que le sol n'était pas en cause et qu'il s'agissait de simples fissures dues à des variations dimensionnelles d'un plancher béton dans lequel étaient incorporées les canalisations du chauffage central par eau chaude.

L'expert judiciaire a donc prescrit des travaux confortatifs, coûteux, totalement inutiles, alors qu'un géotechnicien, même peu expérimenté, aurait pu lui prouver qu'il ne s'agissait pas de fissures imputables à un tassement des couches porteuses et, qu'en conséquence, des travaux d'injection seraient absolument inefficaces.

\subsection{Glissement de terrain. Effondrement d'un mur de soutènement}

Un particulier a fait récemment construire une maison individuelle dans la région Rhône-Alpes par une grande société renommée, spécialisée dans la livraison de villas clefs en main. Le terrain était en légère déclivité, environ $10^{\circ}$, êt il était notoire qu'il se trouvait dans une zone sujette à glissements de terrain.

Le certificat d'urbanisme et le permis de construire avaient attiré l'attention sur ce point. Malgré sela la décision de construire fut prise après qu'un semblant, pour ne pas dire une parodie, de reconnaissances géotechniques ait été exécuté par la société en question, qui était maître d'œuvre, concluant à la faisabilité de la villa.

La villa fut réalisée sur une plate-forme partiellement en remblais et lors de son achèvement, le futur propriétaire s'aperçut, comme s'est bien souvent le cas; que le devis descriptif des travaux était flou et fort discret sur les terrassements à l'arrière de la villa, côté colline.

Finalement, la réalisation d'un talus incliné à $30^{\circ}$ environ fut entreprise. Malgré les remarques du terrassier, petit artisan local, qui pressentait la nécessité d'un soutènement, le promoteur ne réagit pas et dès les premiers terrassements, un glissement de terrain important se produisit. Fort heureusement, ce glissement était superficiel et il fut bloqué sans dommages par la villa.

Un architecte fut désigné comme expert par le Tribunal de Grande Instance du lieu, avec en particulier, 
comme mission de préconiser les travaux de réfection. Sans qu'aucun essai géotechnique ait été exécuté, son rapport concluait à la nécessité d'exécuter un mur de soutènement en béton armé à réaliser en trois phases successives avec une tranchée drainante à l'amont du terrain. Toutefois, ce rapport était malheureusement vague et imprécis sur le dimensionnement du mur et les conditions d'exécution des travaux.

Suite à cette expertise, une entreprise, connue pour son sérieux dans la région, a été retenue par le demandeur et lors des premiers coups de pelle pour le terrassement du mur de soutènement, un nouveau glissement de terrain se produisit, plus important que le précédent, intéressant une superficie d'environ $750 \mathrm{~m}^{2}$, miraculeusement sans dommages importants pour la structure de la villa qui se retrouvait, cependant, avec une hauteur de terre dépassant de beaucoup les allèges de sa façade arrière. Il fut heureux que ce glissement se soit déclenché avant la construction du mur de soutènement en béton car celui-ci calculé pour une poussée des terres classique avec angle de frottement interne de $30^{\circ}$, aurait, sans l'ombre d'un doute, été entraîné par le terrain contre la villa.

L'un de nos amis géotechnicien confirmé fut alors désigné, par le Tribunal de Grande Instance saisi à nouveau, en qualité de second expert conjointement avec l'expert-architecte précédent. La nouvelle solution préconisée fut l'exécution, dans une première phase, d'une tranchée drainante transversale et de deux éperons drainants dans le sens de la pente du terrain, leur profondeur fut déterminée par l'exécution d'un puits de reconnaissance lors de la réalisation des fouilles qui permit de localiser le plan de glissement à $2,20 \mathrm{~m}$ de profondeur (couche de lignite avec venues d'eau entre une couche supérieure d'argile plastique et une couche de marne raide).

Le géotechnicien préconisa le prélèvement d'échantillons de sols et l'exécution d'essais de laboratoire afin de dimensionner un soutènement en gabions à exécuter par tronçons au cours d'une deuxième phase des travaux confortatifs. Dans ce cas un tel ouvrage est moins couteux et plus sûr qu'un mur de soutènement en béton car il est perméable et susceptible de s'adapter aux mouvements éventuels du terrain. Il a été prescrit également, que le dimensionnement définitif du soutènement et le contrôle technique de son exécution soient confiés à un ingénieur-conseil compétent en mécanique des sols, ce qui fut fait. La stabilisation du terrain est aujourd'hui achevée à la satisfaction de toutes les parties.

\subsection{Cas de la villa de madame $\mathrm{A}$}

Cette villa a été construite dans le département du Rhône, début 1969 par l'entreprise de maçonnerie T. d'après les plans du maître d'œuvre $M$. L'occupation des lieux et la réception tacite remontent à août 1969. Jusqu'au printemps 1976, aucun désordre n'est observé, puis apparaissent à ce moment là des taches d'humidité très importantes sur les murs nord et ouest de la villa et dans la moquette de la chambre à coucher. Cette humidité persistant pendant plus d'un an, la propriétaire Mme $\mathrm{A}$. intente un procès à son voisin $R$., car le maçon $T$. et le maitre d'ouvrage $M$. attribuent ces venues d'humidité au comblement en 1974 d'une mare par R. située sur son terrain, à une trentaine de mètres de la villa $\mathrm{A}$.

Désigné par ordonnance de référé du 8 juin 1977 du T.G.I. local, un premier expert B., rejoignant l'idée émise par M., par $T$. et par l'expert $X$. de la compagnie d'assurances de T., dépose un rapport très succinct le 18 août 1977 concluant que l'humidité provenait du comblement en 1974 de la mare appartenant à $\mathrm{R}$. (alors que pendant deux ans après ce comblement, aucune humidité n'était apparue).

A la suite du dépôt de ce rapport d'expertise, la chambre à coucher de Madame $A$. très humide, a été entièrement refaite avec pose de papiers peints sur des plaques de plomb (!) de $1,50 \mathrm{~m}$ de haut sur la périphérie des murs extérieurs et intérieurs et mise en place d'une nouvelle moquette.

Hélas, ces réfections se révélèrent tout à fait inefficaces, l'humidité persistant, la moquette restant gorgée d'eau. Nous avons alors été désigné comme nouvel expert par le T.G.I. Immédiatement, nous avons fait procéder à des sondages dans le plancher et dans le mur mitoyen, ce qui n'avait pas été réalisé lors de la première expertise. Le plancher constitué de hourdis de béton creux était posé directement sur une couche de graviers répandue sur le sol naturel. Ces hourdis étaient recouverts d'une forme de béton recevant une chappe en ciment sur laquelle avaient êté posés soit les carrelages des W.C., hall et salle de bains, soit la moquette de la chambre à coucher (fig. 1).

Dans les trous de sondages, nous constatons qu'il existe des stalactites blanches à travers le plancher, ce qui prouve d'une manière formelle que l'eau s'écoule du haut vers le bas et ne remonte pas du bas vers le haut, malgré les affirmations de M., de T., de X. et du premier expert judiciaire B. (fig. 2).

Nous vérifions immédiatement les raccords des W.C. et salle de bains réalisés par le plombier V. Il apparaît aussitôt qu'il y a eu des malfaçons dans leur exécution, d'oũ des fuites au niveau du plancher. Nous faisons, rapidement, procéder aux réparations de ces joints défectueux, mais malgré cela, l'humidité dans le plancher de la chambre à coucher subsiste.

Alors, M. et l'expert X. de la compagnie d'assurances de T. persistant à vouloir que cette humidité, génératrice des désordres, soit imputable uniquement au comblement de la mare R., proposent d'effectuer un drainage périphérique aux frais de la compagnie d'assurances de $T$. Bien que nous ne soyons pas convaincu de l'efficacité de ces travaux de drainage, comme ils ne coûtent rien à la demanderesse, nous acceptons qu'ils soient réalisés, pour montrer à $\mathrm{M}$., T. et $\mathrm{X}$. que leur opinion est erronée.

Le drain mis en place débite légèrement, et au début mars 1979 . M., T. et X triomphent, car un assèchement semble s'être produit. Ce triomphe est de courte durée, car en août 1979, il est constaté, à nouveau, 


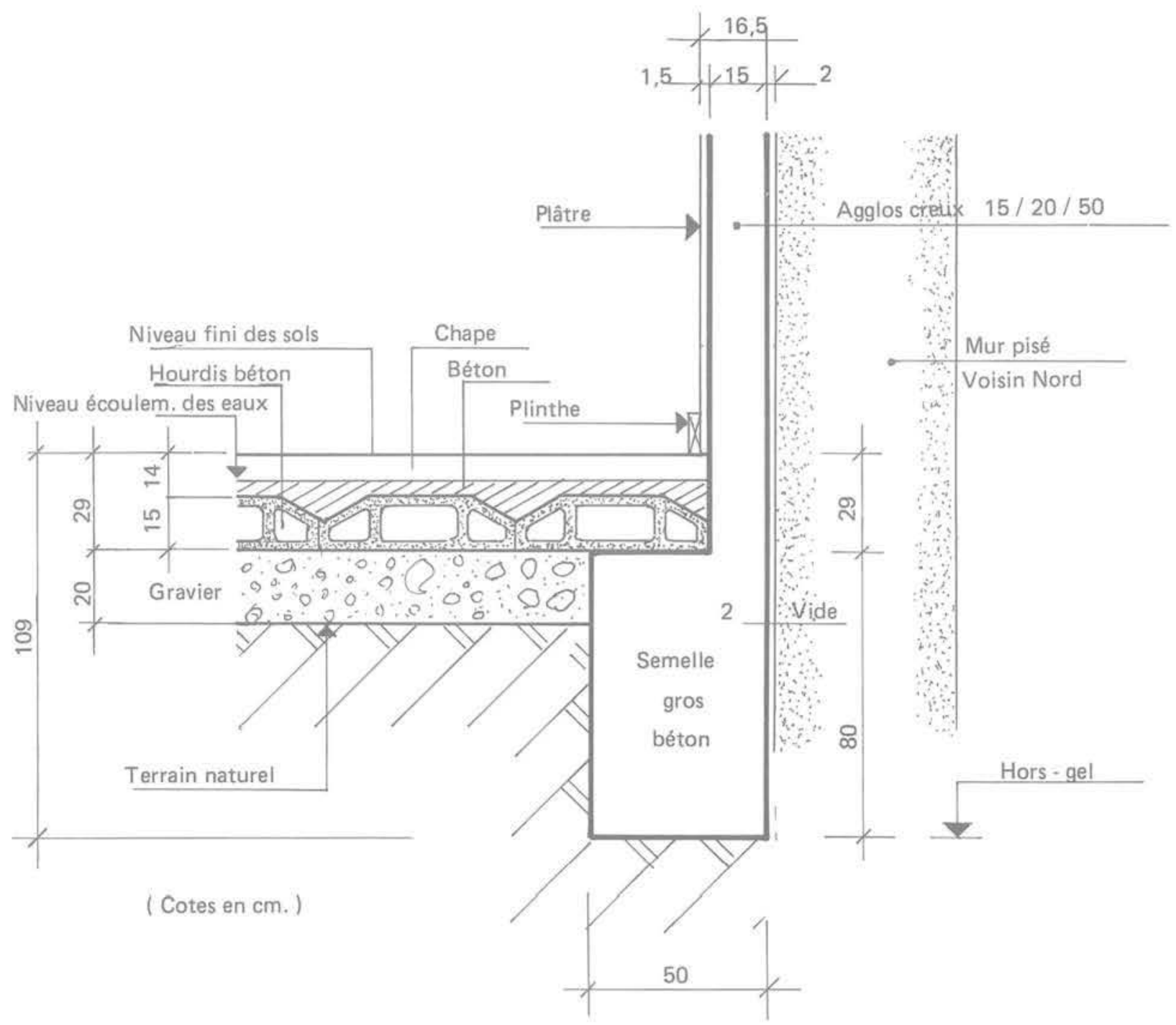

Fig. 1. - Coupe plancher sur terre-plein de la chambre de $M m e$ A.

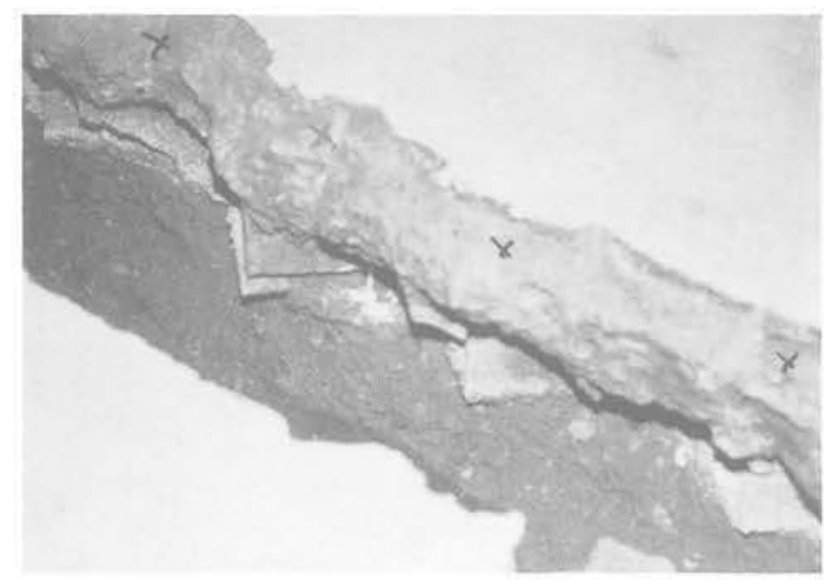

Fig. 2. - Vue latérale de la tranchée réalisée dans le plancher de la chambre de Mme A. lles croix indiquent le niveau des venues d'eau s'écoulant à la base de la chape sur la dalle béton. Remarquer la stalactite blanche). que l'humidité de la chambre à coucher de Mme A. s'est aggravée. De l'eau continue à tomber goutte à goutte le long de stalactites visibles dans la tranchée réalisée au travers du plancher de cette chambre (fig. 2).

Bien que nous ayons insisté à différentes reprises pour obtenir de $\mathrm{V}$. et de $\mathrm{M}$. le plan détaillé des canalisations encastrées dans la chape béton supportant le carrelage de la salle de bains, des W.C., du hall et de la cuisine, tant pour l'alimentation en eaux que pour les évacuations d'eau des différents appareils sanitaires, celui-ci ne peut nous être fourni. Un essai de mise en pression des canalisations semble montrer que ces dernières sont étanches. Mais comme nous n'avons pas pu obtenir le plan des canalisations, il se peut que toutes n'aient pas été contrôlées. En conséquence, nous exigeons la mise hors service de toutes les canalisations encastrées dans le sol et leur remplacement provisoire par des canalisations apparentes. Cette modification du réseau des canalisations d'alimentation des eaux fait cesser immédiatement les désordres. En quelques 
jours, l'assèchement de la chambre de Mme A. survient enfin.

Cela démontre que l'humidité observée dans le plancher et à la base des murs de la villa de Mme A. provenait d'une fuite résultant de la corrosion d'une canalisation sous pression encastrée dans la chape béton supportant le carrelage ou la moquette.

Cela prouve péremptoirement, comme nous l'avions affirmé dès notre premier accédit, que le comblement de la mare de $\mathrm{R}$. n'avait rien à voir avec l'humidité de la chambre à coucher de Mme A., ce qui était évident a priori au point de vue mécanique des sols.

Nous voyons là un exemple caractéristique où expert judiciaire, entrepreneurs, expert d'une compagnie d'assurances et maître d'œuvre se sont cristallisés sur une idée totalement fausse. Ils se sont contentés d'affirmer conjointement que leur idée était la bonne sans vouloir rechercher la cause réelle des désordres qui, manifestement, n'étaient pas imputables à un problème de mécanique des sols lié à des remontées d'eau en provenance du terrain voisin.

Ces experts ont entraîné la compagnie d'assurances de $T$. à payer des drains totalement inutiles sans résoudre le problème posé.

Naturellement, le réseau des canalisations installés provisoirement en apparent pour les nécessités de l'expertise, a été transformé ensuite en définitif.

Précisons que le tribunal a entériné notre rapport, mis hors de cause $\mathrm{R}$. et sa mare qui n'était pour rien dans le sinistre, condamné l'entreprise $V$. aux réfections et également, Mme A. à payer $7500 \mathrm{~F}$ à $\mathrm{R}$. à titre de dommages et intérêts pour instance abusive.

\subsection{Effondrement d'un immeuble dû à une explosion de gaz}

Le cas que nous allons résumer ci-dessous est très caractéristique d'une erreur commise par trois experts judiciaires et des conséquences graves que cela aurait pu entraîner.

Le 7 janvier 1971, une violente explosion de gaz se produit dans un immeuble ancien de la ville de D., bientôt suivie d'un incendie. L'immeuble ancien composé de trois niveaux sur rez-de-chaussée s'effondre totalement, ce qui a entraîné de très nombreux blessés plus ou moins gravement atteints et la mort de deux personnes.

Trois experts sont désignés par le tribunal, un chimiste, un ingénieur, un industriel. Ils procèdent à leur enquête et éliminent, à juste titre, une explosion due à des explosifs, Compte tenu du type de déflagration, ils arrivent à la conclusion qu'il s'agit d'une explosion d'une fuite de gaz provenant d'une canalisation extérieure parallèle à l'immeuble et proche de celui-ci (fig. 3).

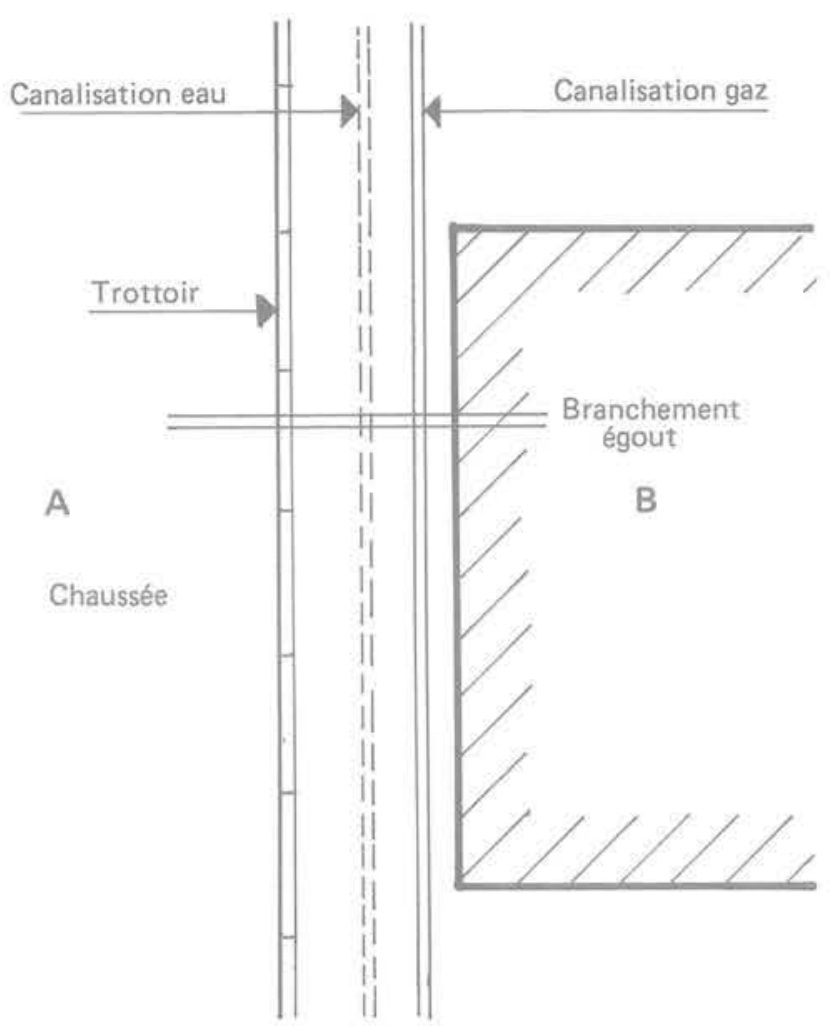

Fig. 3. - Implantation de la canalisation gaz litigieuse.

Il s'agissait d'une conduite de gaz posée dans les années 1925-1927. Les experts ont estimé que la fuite résultait des travaux réalisés par l'entreprise L. le 22 août 1957, pour mettre en place un branchement d'égouts desservant le bâtiment, installé perpendiculairement à la canalisation de gaz. La tranchée faite pour ce raccordement d'égouts avait $60 \mathrm{~cm}$ de large seulement et passait sous la canalisation de gaz (fig. 4). Les experts ont reproché à L. d'avoir remblayé sa tranchée avec de la terre végétale noire, généralement compressible, d'où, disaient-ils, des tassements ayant entraîné la déformation de la canalisation de gaz, ses fissurations et la fuite. Or, un voisin avait signalé aux experts que d'autres travaux avaient été réalisés fin 1970 dans la rue et qu'à cette occasion, à plusieurs reprises, des odeurs de gaz avaient été perçues et qu'après la réfection des trottoirs recouverts d'un revêtement bitumineux on n'avait plus décelé d'odeurs de gaz. Les trois experts n'ont tenu aucun compte de cette information pourtant très importante.

A la suite du dépôt du rapport des trois experts, le juge d'instruction a procédé à l'inculpation de l'entrepeneur $L$. qui a été condamné par la suite à 4 mois de prison avec sursis et à $5000 \mathrm{~F}$ d'amende par jugement du 29 janvier 1972 du tribunal correctionnel de D.

Un de nos amis, géotechnicien qualifié, ayant étudié le problème est arrivé à la conclusion que la fuite de la canalisation gaz ne pouvait être imputée au terrassement de la petite tranchée pour le branchement 


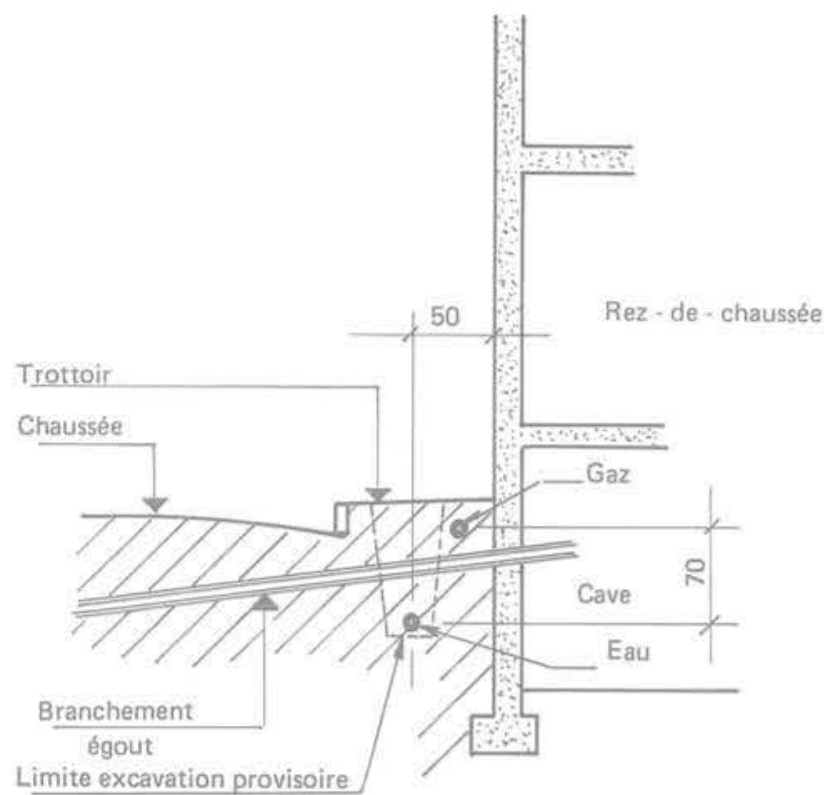

Fig. 4. - Coupe AB. Fouille provisoire exécutée quelques semaines avant l'explosion.

d'égouts perpendiculaire à la conduite gaz effectué par L. le 22 août 1957, mais, bien au contraire, aux travaux de pose d'une canalisation d'eau parallèle à la canalisation gaz sur une très grande longueur, à $30 \mathrm{~cm}$ seulement de cette dernière et située à $70 \mathrm{~cm}$ en contre-bas, effectués quelques semaines avant l'explosion par une autre entreprise X (fig 4). Ces travaux ayant inévitablement décomprimé le sol sur une très grande longueur, étaient certainement à l'origine des déformations de la canalisation gaz. Il y a donc eu appel. Par arrêt du 30 octobre 1974, la cour d'appel de D. a désigné deux nouveaux experts dont les conclusions ont été très différentes de celles de leurs trois premiers collègues. En effet, ces deux nouveaux experts ont reconnu le bien fondé des observations de notre ami géotechnicien. En outre, en poursuivant leurs investigations, ils ont montré, de plus, que la pression de distribution du gaz manufacturé était autrefois de $80 \mathrm{~mm}$ d'eau alors que celle du gaz naturel distribué depuis 1963 était de $200 \mathrm{~mm}$ d'eau. Cela a pu naturellement accroître les fuites, d'autant plus qu'il est apparu que le nouveau gaz avait des propriétés très desséchantes, ce qui expliquait la dessication des joints de la canalisation et les premières fuites ainsi que la coloration noirâtre des terres prises à tort par les premiers experts pour des terres végétales.

Sur la base du rapport très détaillé des deux nouveaux experts, la Cour a relaxé l'entreprise $\mathrm{L}$. sans peine ni dépends.

On voit là, l'importance d'une expertise judiciaire correctement conduite. $\mathrm{Si}$ parmi les trois premiers experts, il y avait eu un géotechnicien, il aurait pu montrer que les travaux de terrassements de l'entreprise L., réalisés en 1957 n'étaient pour rien dans l'explosion du 7 janvier 1971 et que, par contre, c'étaient les travaux exécutés par l'entreprise $\mathrm{X}$. quelques semaines avant l'explosion qui étaient à l'origine de la déformation de la vieille canalisation gaz.

\subsection{Exemple brésilien - Effondrement ayant provoqué 63 morts}

Il n'y a pas qu'en France où des erreurs d'expertise arrivent. Lors de nos voyages à l'étranger, nous avons pu constater que souvent, l'absence de géotechniciens dans le cours d'une expertise avait pu avoir des conséquences importantes.

Au Brésil, le code civil est différent du nôtre, en particulier, la responsabilité des constructeurs n'est que de 5 ans, et non pas 10 ans. Le régime de l'expertise judiciaire est également différent, mais il y a eu aussi des cas où la méconnaissance de la mécanique des sols a conduit à des erreurs graves. Citons l'exemple d'un immeuble qui s'est effondré en 1972, quinze jours avant son inauguration, entraînant la mort de 63 personnes.

Les premiers experts désignés ont attribué cet effondrement à la conjonction de trois causes dans des proportions égales:

- Erreur de calcul dans les structures;

- Erreur dans la programmation du décoffrage des structures:

- Erreur dans les fondations constituées de pieux qui auraient tassé de 2 à $5 \mathrm{~cm}$.

Or la structure était constituée de piliers espacés de $60 \mathrm{~m}$ avec 4 niveaux de plancher. Un géotechnicien a pu démontrer que les fondations sur pieux n'étaient absolument pas à l'origine du sinistre.

Il a été prouvé que le sinistre était imputable à de très graves erreurs de calcul des poutres de très grande portée, dont le ferraillage était notoirement insuffisant.

Ces différents exemples montrent que le rôle du géotechnicien peut être capital pour la recherche de la cause réelle des désordres liés à la mécanique des sols et aux fondations, donc à la recherche de la vérité.

Naturellement, les parties auront très souvent intérêt à se faire assister par un géotechnicien compétent, particulièrement lorsque l'expert judiciaire n'est pas un géotechnicien.

Au cas où, après un premier jugement, une partie estimerait qu'elle doit faire appel car l'expertise judiciaire n'a pas permis de réunir tous les éléments nécessaires à la compréhension des désordres, il ne faut pas qu'elle hésite à recourir à un géotechnicien pour lui confier une mission d'expertise officieuse. On s'interroge souvent sur la validité et l'utilité d'un tel rapport déposé pour combattre un rapport d'expertise officiel déjà homologué par un tribunal. Il paraît utile à ce sujet de citer un arrêt récent de la cour d'appel de Lyon qui fera certainement jurisprudence.

Il s'agit d'une décision rendue le 21 octobre 1982 par la Cour d'Appel de Lyon dans une affaire Briet contre Arnoldy. 
L'appelante avait produit trois rapports d'expertise officieux.

La Cour a retenu l'intérêt des conclusions techniques contenues dans ces rapports officieux après avoir déclaré dans ses attendus:

"Attendu que les appelants se prévalent pour demander l'infirmation de la décision déférée de rapports officieux émanant de Monsieur Bandier, de l'architecte Guilleret, et de Monsieur de Prandieres... .

«Attendu qu'il est vrai que ces consultations n'ont aucun caractère contradictoire, mais que les époux Briet y puisent des éléments de discussion qui ne peuvent être écartés...

\section{CONCLUSIONS}

Quelle que soit leur discipline, il faut attirer l'attention des experts sur le danger de se prononcer dans un ou des domaines hors de leur spécialité. Il est toujours bénéfique de faire intervenir un spécialiste.

En effet, la responsabilité des experts judiciaires peut être recherchée, non seulement du fait du retard dans les opérations d'expertise ou à la suite de négligences matérielles ou du non respect des règles élémentaires de procédure judiciaire, mais encore en raison des erreurs commises.

Il ne faut pas oublier que la responsabilité des experts est une responsabilité de droit commun fondée sur l'article 1382 du Code civil, c'est-â-dire reposant sur la notion de fautes. Mais quelle est la définition de la faute en cette matière?

A ce sujet, il est utile de rappeler la position prise par le Tribunal Civil de Nîmes dans la fameuse affaire du «Pain maudit * de Pont-Saint-Esprit, qui en a donné le critère suivant, appliqué par la jurisprudence:

«L'expert judiciaire doit être tenu pour responsable de toutes fautes, même légères, que n'aurait pas commises un expert avisé s'il est démontré une relation de cause à effet entre la faute commise et le dommage causé» (Tribunal Civil de Nîmes, $1^{\text {er }}$ juillet 1958 , semaine juridique 1959-11-347).

Ce lien de causalité direct et nécessaire entre la faute et le préjudice a été retenu également par le Tribunal de Grande Instance de Paris, $1^{\text {re }}$ chambre, $1^{\text {re }}$ section, 26 avril 1978, (Gozette du Palais, 1978, 2.449).

Il est évident qu'il s'agit là d'une très lourde responsabilité. Il est donc indispensable que l'expert judiciaire soit bien assuré pour répondre à des mises en cause éventuelles. Nous ne pouvons qu'inciter les experts judiciaires à souscrire une police particulière d'assurance pour faire face à celles-ci. L'expérience montre qu'une police d'assurance groupe, souscrite par l'intermédiaire des compagnies régionales d'experts judiciaires, permet d'obtenir pour des primes modestes, des garanties très complètes.

En ce qui concerne les expertises du bâtiment et des travaux publics, nous souhaitons que nos confrères architectes ou ingénieurs structures pensent aux problèmes que peut poser la mécanique des sols et n'hésitent pas à interroger, et au besoin, à faire intervenir, un géotechnicien ou un géologue qualifié en temps utile.

\section{BIBLIOGRAPHIE}

COSTET, J.; SANGLERAT, G. (1983). - "Cours Pratique de Mécanique des Sols», $3^{e}$ édition, Tome 1: Plasticité et calcul des tassements, Tome 2: Calcul des ouvrages.

SANGLERAT, G. (1980). - $-\propto$ Analyse d'une expertise judiciaire concernant des tassements différentiels de dallages industriels». Revue Technique du Bâtiment et des Constructions Industrielles $n^{\circ} 81$. NovembreDécembre Paris et "Les Cahiers de l'Expertise», Cahier $n^{\circ} 1$, Lyon.

SANGLERAT, G.; SANGLERAT, T.-R. A. (1983). *Quand faire intervenir un géotechnicien dans une expertise judiciaire en Bâtiment et Travaux Publics? „, Les Cahiers de l'Expertise, Compagnie d'Experts près la Cour d'Appel et le Tribunal Administratif de Lyon, Cahier $n^{\circ} 4$, Lyon. 